\title{
A few analytical solutions to the linear Boltzmann transport equation with an application to channeling
}

\section{Citation}

Golovchenko, J. A. 1976. A Few Analytical Solutions to the Linear Boltzmann Transport Equation with an Application to Channeling. Physical Review B 13, no. 11: 4672-4677. doi:10.1103/ physrevb.13.4672.

\section{Published Version}

doi:10.1103/PhysRevB.13.4672

\section{Permanent link}

http://nrs.harvard.edu/urn-3:HUL.InstRepos:29407069

\section{Terms of Use}

This article was downloaded from Harvard University's DASH repository, and is made available under the terms and conditions applicable to Other Posted Material, as set forth at http:// nrs. harvard.edu/urn-3:HUL.InstRepos:dash.current.terms-of-use\#LAA

\section{Share Your Story}

The Harvard community has made this article openly available.

Please share how this access benefits you. Submit a story.

\section{Accessibility}




\title{
A few analytical solutions to the linear Boltzmann transport equation with an application to channeling*
}

\author{
J. A. Golovchenko ${ }^{\dagger}$ \\ Brookhaven National Laboratory, Upton, New York \\ and Bell Laboratories, Murray Hill, New Jersey
}

(Received 19 August 1974)

\begin{abstract}
Some exact solutions to the linear Boltzmann transport equation in a one-dimensional space are presented. These results may be applied to demonstrate the trend towards statistical equilibrium of fast channeled particles in the transverse plane. Statistical mechanics of channeled particle motion is then discussed in terms of the random string approximation and further considerations.
\end{abstract}

\section{INTRODUCTION}

This short note has been stimulated by an interesting recent paper by Lindhard and Nielsen ${ }^{1}$ dealing with the general linear description of statistical systems in physics. In their work several exact solutions of the linear transport equation are presented corresponding to various hypothetical physical systems approach towards equilibrium. By similar methods I have found some further simple analytic solutions. The motivation for this work was to obtain a simple theoretical description of the angular distribution of fast axially channeled particles transmitted through very thin crystals. Some preliminary experimental work in this area has already been reported. ${ }^{2}$

Thus in Sec. II we review the formalism and present the new solutions in terms of the abstract probability field. In Sec. III we apply these results to the transverse momentum distribution function for axially channeled particles. This distribution function can be observed in transmission experiments and is also of interest in backscattering experiments.

\section{MATHEMATICAL FORMALISM}

Consider a probability field $a(x, T)$ whose (linear) equation of motion is

$$
\frac{\partial a(x, T)}{\partial T}=\int \Gamma(x, y, T) a(y, T) d y .
$$

Here $x$ symbolizes the coordinates of the space in which $a(x, T)$ is defined and $T$ is a parameter measuring the evolution of the field. If in addition, it is required that $a(x, T)$ be conserved in the sense that

$$
\int \frac{\partial a}{\partial T}(x, T) d x=0
$$

it follows that Eq. (1) may be rewritten as

$$
\begin{array}{r}
\frac{\partial a}{\partial T}(x, T)=\int d y[G(x, y, T) a(y, T) \\
-G(y, x, T) a(x, T)] .
\end{array}
$$

$G$ must be real and greater than or equal to zero and can be looked upon as a transition rate. The reader is referred to Ref. 1 for a detailed discussion of the general properties that equations of this form have with particular emphasis on problems in statistical physics.

For the application to follow it is satisfactory to consider kernels $G$ having the form

$$
G(x, y, T)=G(|x-y|)
$$

that correspond to time-independent translationally invariant interactions. The detailed functional form of $G$ depends on the nature of the space and the specific interactions considered.

A general solution to Eq. (2) subject to the conditions of Eq. (3) may be constructed by separating the $x$ and $T$ dependence in Eq. (2). Thus

$$
a(x, T)=\sum C_{k} a_{k}(x) f_{k}(T)
$$

The eigenfunctions of the field $a_{k}(x)$ must evidently satisfy the eigenvalue equation

$$
\lambda_{k} a_{k}(x)=\int d y G(x, y)\left[a_{k}(x)-a_{k}(y)\right],
$$

with $\lambda_{k}$ the separation constant (eigenvalue). The translational invariance of $G$ implies that in one dimension

$$
a_{k}(x)=e^{i k x} \text {. }
$$

The allowed $k$ values are determined by the boundary condition to be discussed shortly. It follows that the associated eigenvalue must be

$$
\lambda_{k}=\int d \eta G(\eta)\left(1-e^{i k \eta}\right) .
$$

It also follow that 


$$
f_{k}(T)=e^{-\lambda_{k} T} \text {. }
$$

The values of $C_{k}$ and the allowed values of $k$ are determined by the initial conditions and boundary conditions imposed upon the solution. It would thus appear that the main obstruction to obtaining analy tically useful formulas for $a(x, T)$ is the evaluation of $\lambda_{k}$ from Eq. (7). If possible it would be of great value to perform the sum in Eq. (4). This concludes the review of the recipe for solving Eq. (2) under these specialized conditions.

It is possible to propose some specific forms of $G$ that have relatively simple solutions for $a(x, T)$. In Sec. III it will be shown that these results give a useful insight into the channeling phenomena. First we impose the periodic boundary condition

$$
a(x, T)=a(x+2 \pi, T) .
$$

$x$ thus appears as an angle variable. Therefore,

$$
k=n, n=0, \pm 1, \pm 2 \text {. }
$$

The initial value $a(x, 0)$ is taken to be

$$
a(x, 0)=\delta(x),
$$

$\delta(x)$ being the Dirac $\delta$ function.

Consider now the following forms for $G$ :

\section{Case a}

$$
G=\text { const }=1 \text {. }
$$

Then (7) gives easily

$$
\lambda_{k}=2 \pi\left(1-\delta_{k}\right)
$$

with $\delta_{k}$ being the Kronecker $\delta$ function. Now using the decomposition

$$
\delta(x)=\frac{1}{2 \pi} \sum_{k} e^{i k x}
$$

gives

$$
\begin{aligned}
& a(x, T)=\delta(x) e^{-2 \pi T}+(1 / 2 \pi)\left(1-e^{-2 \pi T}\right) . \\
& \quad \text { Case b } \\
& G=(1 / \pi) \sin ^{-2}\left(\frac{1}{2} x\right) .
\end{aligned}
$$

Using the result that

$$
\frac{1}{\pi} \int_{0}^{2 \pi} d \eta \frac{\sin ^{2}\left(\frac{1}{2} k \eta\right)}{\sin ^{2} \frac{1}{2} \eta}=|k| .
$$

Equation (7) gives

$$
\lambda_{k}=|k| \text {. }
$$

Then using

$$
\sum_{n} x^{n}=\frac{1}{1-x}
$$

one can perform the sum in (4) giving

$$
a(x, T)=\frac{1}{2 \pi} \frac{\sinh T}{\cosh T-\cos x}
$$

with $\sinh x$ and $\cosh x$ being the hyperbolic functions.

$$
G=\pi /\left[\pi^{2}-(\pi-|x|)^{2}\right]^{3 / 2} .
$$

$\lambda_{k}$ may be evaluated from (7) with the substitution $1-x / \pi=\sin \varphi$ and integration by parts, giving

$$
\lambda_{k}=-\pi k \cos k \pi J_{1}(k \pi) \text {. }
$$

For this case I've been unable to sum (4) and thus leave the solution as

$$
a(x, t)=\frac{1}{2 \pi} \sum_{k} e^{i k x} e^{\pi_{k}} \cos k \pi J_{1}(k \pi) T .
$$

These three solutions form the basis for our discussion of channeling to follow.

\section{APPLICATION TO CHANNELING}

It has been recognized for about ten years now that fast particles (i.e., a few MeV protons or alpha particles) penetrating crystalline materials may, under certain circumstances undergo series of strongly correlated collision sequences with atoms of the target crystal.

A manifestation of this possibility occurs when considering penetration along a low-index axial direction in the crystal. If one imagines a positive particle whose classical path lies between and makes a small angle with the rows of atoms making up the low-index axial direction, its trajectory is determined by gentle collision sequences whose net effect is to steer the particle away from smallimpact-parameter collisions with the solid's atoms. This state of motion is called axial channeling.

The problem of predicting the particle trajectories and flux in the crystal during the channeling condition has been approached seriously in two ways, both based upon a classical view of the motion. The first method involves the computer simulation of compound binary collisions of the penetrating particle with the atoms in the crystal. This method has been successful in predicting the outcome of various channeling laboratory experiments. An alternative approach based upon the methods of analysis is that of Lindhard. ${ }^{3}$ This work has provided a simple picture of the channeling process of which we shall attempt to take advantage. (For an interesting comparison of the two approaches see Ref. 4.)

By this method it is shown that a good approxi- 
mation to the channeled trajectory is obtained by replacing the strings of atoms along the low index direction by a continuous rod of potential

$$
U(r)=\frac{1}{d} \int V\left[\left(r^{2}+z^{2}\right)^{1 / 2}\right] d z .
$$

Here $V$ is the potential-energy function associated with the ion-atom interaction, $r$ is the distance from the string, and $d$ is the atomic spacing along the string whose extension lies in the $z$ direction. Thus, in this approximation, one need consider the motion projected on a plane perpendicular to the axial direction, the velocity along the $z$ direction remaining constant.

Let a particle be at a distance $r$ from the string and be traveling at an angle $\psi$ relative to it. If the particle has an energy $E=1 / 2 m v^{2}$, it will have a transverse energy $E_{\perp}=E \sin ^{2} \psi+U(r)$. When $E_{\perp} \gtrless 2 Z_{1} Z_{2} e^{2} / d$ the approximation breaks down and the channeling disappears. $Z_{1}, Z_{2}$ are the atomic numbers of the projectile and host, respectively. Typical angles $\psi$ corresponding to $E_{\perp} \lesssim 2 Z_{1} Z_{2} e^{2} / d$ are $\sim 10^{-2} \mathrm{rad}$ or less.

We now ask for the scattering angle $\theta$ in the transverse plane that a channeled particle undergoes when scattering off a string. Evidently in the continuum approximation described above, the standard solution to this problem is applicable, i.e.,

$$
\theta=\pi-2 b \int_{r_{0}}^{\infty} \frac{d r}{r^{2}}\left(1-\frac{U(r)}{E_{\perp}}-\frac{b^{2}}{r^{2}}\right)^{-1 / 2} .
$$

Here $b$ is the impact parameter with the string and $r_{0}$ is defined implicitly by

$$
1-\frac{U\left(r_{0}\right)}{E_{\perp}}-\frac{b^{2}}{r_{0}^{2}}=0 .
$$

It is now possible to proceed along the lines outlined in Sec. II only if the transverse plane is assumed to consist of randomly distributed strings (random-string approximation). We take this approach and discuss its shortcomings later. Thus it follows that the rate a particle is scattered through an angle $\theta$ by a collision with a string is

$$
P(\theta)=N_{s} v_{\perp}\left|\frac{d b(\theta)}{d \theta}\right|,
$$

where $N_{s}$ is the number of strings per unit area in the transverse plane, $b(\theta)$ is obtained by the inversion of Eq. (15), and $v_{\perp}$ is the transverse velocity far from the strings (i.e., $E_{\perp}=\frac{1}{2} m v_{\perp}^{2}$ ).

A transport equation for the probability of finding a particle at $\theta$ can now be constructed in the form (2).

$$
\frac{\partial a(\theta, t)}{\partial t}=N v_{\perp} \int_{-\pi}^{\pi} d \theta^{1} \frac{d b\left(\theta^{1}\right)}{d \theta^{1}}\left[a\left(\theta-\theta^{1}\right)-a(\theta)\right],
$$

where $t$ is the time of evolution. Observe that this distribution function $a(\theta)$ is directly observable in experiments where the incident beam of particles is transmitted through very thin crystals. The transverse energy of the particles inside the crystal will be essentially $E \Psi^{2}$, where $\Psi$ is the angle between the crystal axis and the beam direction. The intensity of the transmitted beam as a function of azimuth is $a\left(\theta, t_{0}\right)$ where $t_{0}$ corresponds to the time of emission from the back of the sample. At $t=0$ (when the beam enters the crystal), the beam intensity as a function of azimuth is $a(\theta, 0)=\delta(\theta)$, see Fig. 1 .

Consider some models for the string:

(a) First, for the purpose of a simple illustration, consider the unphysical string interaction that would result in a uniform distribution of scattering angles. It follows that the considerations leading to Eq. 11(a), 12(a), and 13(a) apply if we interpret

$$
T=N_{s} v_{\perp} \sigma_{s} t
$$

with $\sigma_{s}$, the total cross section,

$$
\sigma_{s}=\int_{-\pi}^{\pi} \frac{d b}{d \theta} d \theta
$$

(b) Consider next the potential function

$$
U(r)=\frac{Z_{1} Z_{2} e^{2}}{d} \frac{\pi a_{\mathrm{TE}}}{2 r}
$$

with $a_{\mathrm{TF}}$ the Thomas-Fermi screening length for the $\left(Z_{1}, Z_{2}\right)$ pair defined by

$$
a_{\mathrm{TF}}=0.885 a_{0}\left(Z_{1}^{2 / 3}+Z_{2}^{2 / 3}\right)^{-1 / 2} .
$$

This potential function was used by Lindhard ${ }^{3}$ to estimate the depth in the crystal required to

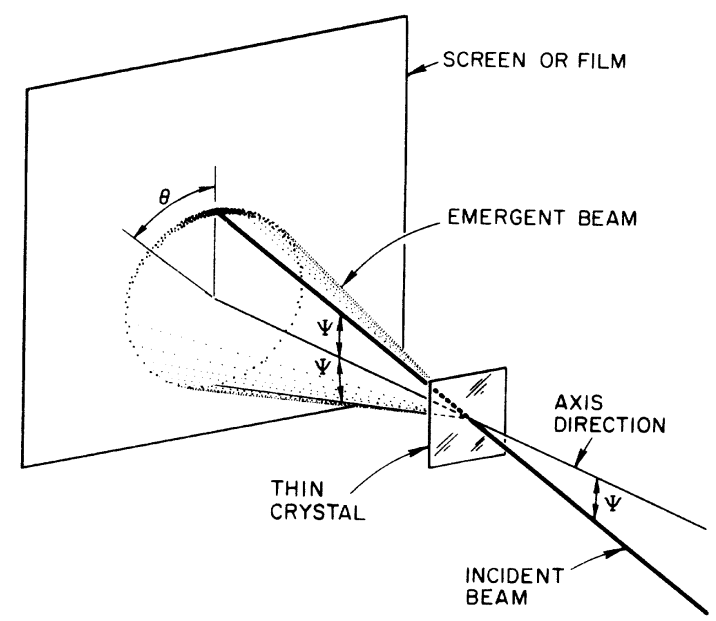

FIG. 1. Schematic illustration of experimental arrangement for observing the distribution discussed in the text. 
achieve statistical equilibrium by a mean-freepath argument. Inserting (20b) in (15) and using (17) gives

$$
P(\theta)=N_{s} v_{\perp} a_{\mathrm{TF}} \frac{Z_{1} Z_{2} e^{2}}{d} \frac{\pi}{8} \frac{1}{E_{\perp} \sin ^{2}\left(\frac{1}{2} \theta\right)} .
$$

It can then be seen that Eqs. (11b), (12b), and (13b) apply if

$$
T=N_{s} v_{\perp} a_{\mathrm{T} \mathrm{F}} \frac{Z_{1} Z_{2} e^{2}}{E_{\perp} d} \frac{\pi^{2}}{2} t
$$

(c) Finally consider

$$
U(r)=\frac{Z_{1} Z_{2} e^{2}}{d}\left(\frac{c a_{\mathrm{TE}}}{r}\right)^{2}
$$

where $c$ is a constant $(\sim \sqrt{3})$. This gives

$$
\begin{aligned}
P(\theta)= & N_{s} v_{\perp} a_{\mathrm{TF}} c\left(\frac{Z_{1} Z_{2} e^{2}}{d E_{\perp}}\right)^{1 / 2} \\
& \times \frac{1}{\pi} 1 /\left[1-(1-\theta / \pi)^{2}\right]^{3 / 2}
\end{aligned}
$$

so that (11c), (12c), and (13c) apply when one takes

$$
T=N_{s} v_{\perp} a_{\mathrm{TF}} c\left(Z_{1} Z_{2} e^{2} / d E_{\perp}\right)^{1 / 2} \pi t .
$$

The potential-energy function gives in (20c) is closely related to the Lindhard standard potential ${ }^{3}$ which is

$$
U(r)=\frac{Z_{1} Z_{2} e^{2}}{d} \ln \left[\left(\frac{c a_{\mathrm{TE}}}{r}\right)^{2}+1\right] .
$$

Results for cases $b$ and $c$ are plotted in Figs. 2(a) and 2(b) and they display graphically the trend towards equilibrium in the transverse plane. Note that the transverse energy dependence is incorporated totally in the time scale. Indeed the cases $a, b$, and $c$ illustrate how widely different transverse energy dependencies result from different assumptions about the scattering field. This fact would seem to be quite useful experimentally since observing the transverse energy dependence of the transmitted beams's angular distribution involves only changing the crystal orientation angle $\Psi$.

\section{DISCUSSIONS AND CONCLUSIONS}

The content of Sec. III indicates that simple estimates for the azimuthal dependence of transmitted axial channeled particles can be made based upon some reasonable model string potentials. Experimentally azimuthal distributions are not trivial to measure and it might be hoped that the foregoing analysis will stimulate quantitative work along the lines. Secondly we note that the model solutions obtained correspond only qualitatively to accurate atomic potentials. Nevertheless they
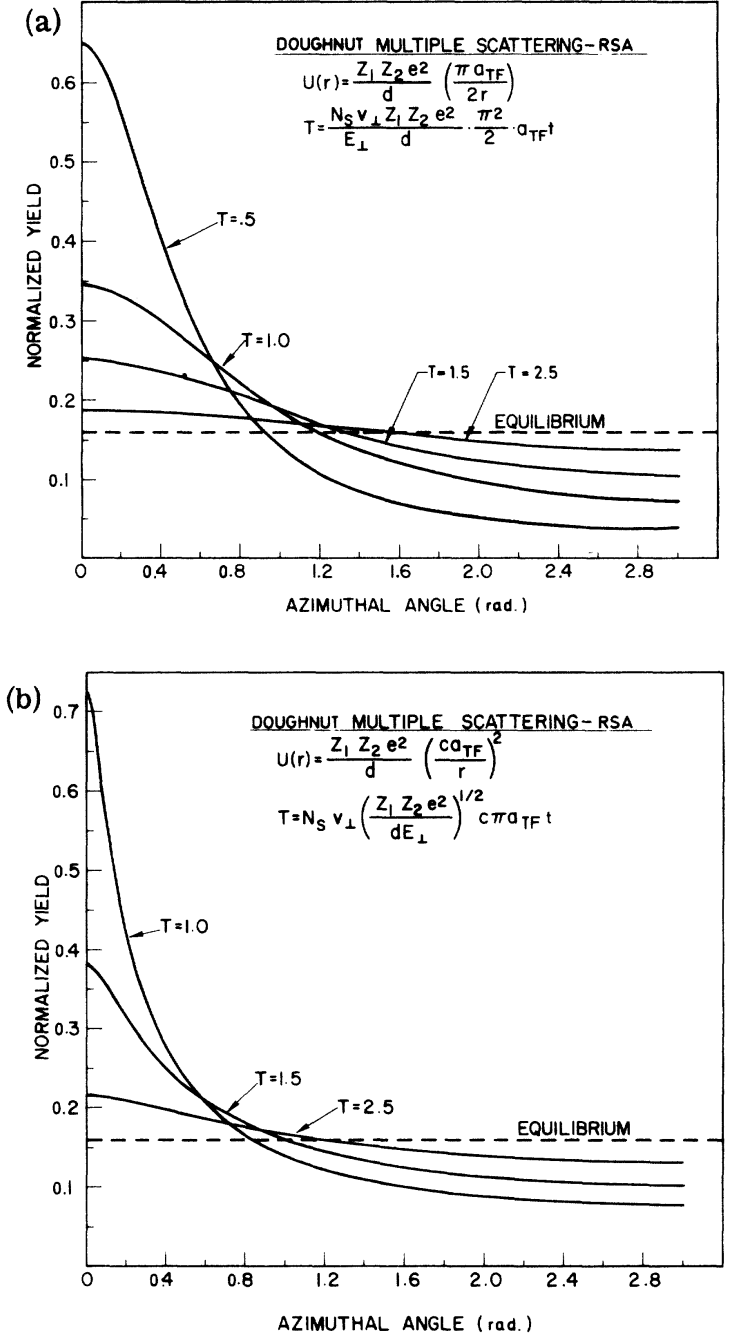

FIG. 2. (a) Angular distribution function corresponding to the $1 / r$ potential function given in Eq. (20b), for several values of $T$. Equilibrium is $T=\infty$. (b) Angular distribution function corresponding to the $1 / r^{2}$ potential function given in Eq. (20c).

are exact solutions and, under suitable stimulation, they can be used in conjunction with perturbation methods to solve Eq. (18) for "hearby" more realistic potentials.

The main approximation in the calculation is the assumption of random distribution of strings in the transverse plane, which ensured invariance under translations. More concretely, for the application at hand, this corresponds to assuming that successive collisions with strings are uncorrelated. Thus the periodic arrangement of strings in the transverse plane suggests that the approximation may introduce serious limitations. After all, periodicity is responsible for the strong correlated scattering that results in axial channeling to begin with. The correlation in that case is most 
neatly described by the existence of the conserved quantity $E_{\perp}$ under the special condition that $E_{\perp} \lessgtr 2 Z_{1} Z_{2} e^{2} / d$. We may anticipate that under certain conditions similar stable trajectories in the transverse motion may develop. Two examples may be noted. The first occurs when the transverse energy is so small that particles are trapped and constrained to move in bound orbits. This condition can arise for both positive and negative particles. For the former case the terms proper or hyperchanneling have been applied and of the latter case the rosette motion ${ }^{5}$ is an example. This condition clearly represents strong correlation; indeed other constants of motion may exist (for example, transverse angular momentum), and the previous calculation totally fails. The second example may be realized for larger transverse energies where trapping is no longer possible but one may imagine channeling due to "strings of strings"3; i.e., simultaneous axial and planar channeling. Here again the strong correlation in collisions is representable by an additional constant of motion, the planar transverse energy. Under such conditions the model of course again fails.

An example of the above may be observed in the pictorial data of Ref. 2, Fig. (1) which shows the transverse momentum distributions of $4-\mathrm{MeV}$ protons transmitted through a $0.51-\mu \mathrm{m}$ silicon crystal near the $\langle 100\rangle$ axial direction. The azimuthal direction of the beam, as measured around the axis direction, was directly along one of the (100) planes. Clearly at the largest tilt angles shown (which still lie within the axial critical angle) a substantial fraction of the beam has been trapped into planar transverse motions.

For strong string directions, which are the most interesting experimentally, the importance of both of these situations is minimized (for positive particles) due to the large distances between strings in the transverse plane [see Fig. (3) of Ref. 1]. That is, the binding energy for proper channeling and the transverse energies for planar channeling are quite small and thus initial conditions corresponding to these possibilities represent a small part of the total transverse phase space available to the axially channeled particle. Experiments must of course not pick out these conditions, for example, by scanning through an axis along a planar direction, if they are to be compared with results from the random string approximation.

With the above considerations and limitations in mind it might be useful to mention a few words about the applicability of the two cases b and $c$ discussed in Sec. III. For trajectories of high transverse energy and small impact parameter $\left(\sim a_{\mathrm{TF}}\right)$, the $1 / r$ potential of case $\mathrm{b}$ probably best describes the scattering, wheras for all impact parameters at smaller transverse energies the $1 / r^{2}$ potential of case $c$ is more appropriate. Thus for distributions far from equilibrium the results of transmission experiments would over all be best described by case $c$.

A free dimensionless time for equilibrium may be taken as $1 / \lambda_{1}$. It follows that crystal thickness $L$ of interest for studies of the trend towards equilibrium can be deduced by setting $t=L / v$ in Eqs. (19), (22b), and (22c) with $T<1 / \lambda_{1}$. It may be noted that the equilibration times for the $1 / r$ and $1 / r^{2}$ potentials roughly agree at large transverse energies and based on our previous discussion, we therefore conclude that a reasonable estimate for all transverse energies may be obtained from the $1 / r^{2}$ potential of case $c$. This suggests that crystal depths required for equilibration are almost independent of transverse energy.

Up until this point the main emphasis has been placed upon transmission experiments where one measures the transverse momentum distribution of the channeled particles. Backscattering experiments on the other hand depend more crucially upon the transverse spatial distributions. Nevertheless, it is possible to draw some conclusions in this case also. It is well known that the major attraction of the assumption of statistical equilibrium lies in the predicted simple spatial flux distributions of channeled particles in the transverse plane. These distributions make possible the simple calculation of backscattering angular yields as a function of the position of the scatterer in the transverse space of the crystal. Indeed, such backscattering experiments have turned out to be one of the most important applications of channeling. However, often this spatial flux information is required at scatterers near the front surface of a crystal, i.e., implanted impurities at a depth at a few hundred angstroms or less. Simple calculations show that equilibrium is not achieved for many practical cases. It might be worth a note at this point that statistical equilibrium is a possible property associated with the four-dimensional transverse phase space. The distributions deduced in this paper may be looked upon as applying to the transverse momentum, $\theta$ measuring the direction of its associated vector. It follows that if the momentum component is not in equilibrium, the distribution in phase is also not and it is therefore not strictly proper under such conditions to calculate the distribution in space from the equilibrium phase distribution. It is difficult to say, though, how serious an error one will make using the equilibrium recipe. Actually arguments not based upon statistical concepts may be invoked that suggest that these er- 
rors may not be very serious. ${ }^{6}$

Recently Barrett ${ }^{7}$ published a note proclaiming the "break down" of statistical equilibrium based upon analysis of computer Monte Carlo calculations. It was observed that large transverse energy particles originating from near string positions underwent focusing collision sequences that led to an anomalously large flux of particles near to strings. Barrett further reasoned that since hard collisions with strings will lead to such focusing collisions at any depth, equilibrium will never be achieved. It would seem that what was actually observed is better described as a limitation of the randomstring approximation and contrary to the implication of the above-mentioned letter, statistical concepts are particularly applicable to the observed effects. To do so we return to our discussion on the effect of simultaneous axial and planar channeling. We assume that such trajectories may be characterized by both an axial and planar transverse energy. It follows from Hamilton's equation that the transverse axial phase space is divided into noncommunicating regions of planar and nonplanar trajectories. Statistical equilibrium in the random string approximation corresponds to assuming uniform probability in phase for both regions. As is well known, this procedure results in a transverse spatial distribution uniform in the accessible space for a given axial transverse energy. ${ }^{3}$ The trajectories considered by Barrett all correspond to initial conditions for nonplanar axial motion. The region of phase space associated with planar-channeled motion therefore should not be included in any averages over phase. On the axial transverse energy shell the equilibrium spatial distribution from all such trajectories with conserved planar transverse energy has a maximum at the center of the corresponding plane and a minimum on the plane. It follows that for the total phase space average to be uniform for a given transverse energy the nonplanar component must compensate and have higher probabilities near the strings. Indeed, we may conclude that the effects should be particularly large when a large region of phase space is occupied by planarchanneled motions, i.e., for weak axes formed from strong planes.

\section{ACKNOWLEDGMENTS}

The author would like to express his gratitude to J. U. Anderson and Vibeke Nielsen of Aarhus University and J. A. Morrison of Bell Laboratories for useful suggestions and discussions.
${ }^{*}$ Research supported in part by the U. S. Atomic Energy Commission.

$\dagger$ Permanent address: Institute of Physics, Aarhus University, DK 8000 , Aarhus C, Denmark.

${ }^{1} \mathrm{~J}$. Lindhard and Vibeke Nielsen, K. Dan. Vidensk. Selsk. Mat.-Fys. Medd. 38, 9 (1971).

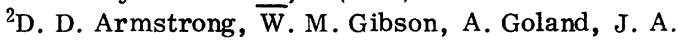

Golovchenko, R. A. Levesque, R. L. Meek, and H. E. Wegner, Radiat. Eff. 12, 143 (1972).
${ }^{3}$ J. Lindhard, K. Dan. Vidensk. Selsk. Mat.-Fys. Medd. 34,14 (1965).

${ }^{4}$ J. U. Andersen and L. C. Feldman, Phys. Rev. B $\underline{1}, 5$ (1970).

${ }^{5}$ H. Kumm, F. Bell, R. Sizmann, and H. J. Kreiner, Radiat. Eff. 12, 53 (1972).

${ }^{6} \mathrm{~J}$. U. Andersen (private communication).

${ }^{7}$ J. H. Barrett, Phys. Rev. Lett. 26, 1542 (1973). 